\title{
Influencia de la simetría facial en la elección de pareja
}

\section{Influence of Facial Symmetry in Mate Choice}

\author{
Katherine Portales Rosas, Carlos Gil Burmann y Marcial Beltrami Boisset ${ }^{l}$
}

\section{Resumen}

Muchos estudios sugieren que la simetría facial, como indicador de estabilidad en el desarrollo y de la salud del individuo, influye en la evaluación de las características de la pareja. Para determinar la importancia de la simetría facial en la elección de pareja, aplicamos, en diversos espacios públicos de Santiago de Chile, una encuesta a 718 personas heterosexuales: 456 mujeres y 262 hombres. Ellos evaluaron tres imágenes de rostros de personas del sexo opuesto con diferentes niveles de simetría facial. Concluimos que la simetría facial influye en la evaluación de las características de una pareja pero, en adición a la simetría, otros factores como el uso de productos de belleza pueden ser señales fenotípicas más importantes que la simetría facial.

Palabras clave: Asimetría fluctuante; elección de pareja; selección sexual.

\begin{abstract}
Many studies suggest that the facial symmetry, as indicator of stability in the development and individual's health, influence the evaluation of the characteristics of the pair. To determine the importance of facial symmetry in mate choice, we have applied questionnaires in diverse public space of Santiago de Chile to 718 heterosexual people: 456 women and 262 men. They had to evaluate three images of faces of people of the opposite sex with different levels of facial symmetry. We concluded that the facial symmetry influences the evaluation of the pair's characteristics but, in addition to the symmetry, other factors like the use of artificial beauty products can be more important phenotypic signals than facial symmetry.
\end{abstract} Key words: Fluctuating asymmetry; mate choice; sexual selection.

1 Katherine Portales Rosas. Departamento de Biología, Universidad Metropolitana de Ciencias de la Educación (UMCE). Licenciatura en Educación y Pedagogía en Biología y Ciencias Naturales. Santiago de Chile Katherine.portales@gmail.com

Carlos Gil Burmann. Departamento de Psicología Biológica y de la Salud, Universidad Autónoma de Madrid (UAM). Biólogo, doctor y profesor titular de Etología y Primatología de la Universidad Autónoma de Madrid (UAM). Madrid, Espańa. cgil@uam.es

Marcial Beltrami Boisset. Zoólogo, etólogo y Académico de la Facultad de Ciencias Sociales de la Universidad de Chile y de la Universidad Metropolitana de Ciencias de la Educación (UMCE) Santiago, Chile. 


\section{Introducción}

Ya hace más de un siglo Darwin propuso en The Descent of Man and Selection in Relation to Sex (1971) que el atractivo físico es un componente biológico importante que aporta información valiosa sobre la potencial pareja. Desde una perspectiva evolutiva actual, los mecanismos psicológicos que evalúan el atractivo físico son adaptaciones que han evolucionado para aumentar el éxito reproductor. Numerosos estudios sugieren que la belleza física es un indicador de la salud y la fecundidad y por ello los individuos atractivos confieren un mayor potencial reproductor a aquellos que los elijen como pareja. La cara es la parte corporal más implicada en el reconocimiento interpersonal y uno de los componentes biológicos más importantes del proceso de elección de pareja. La preferencia por determinadas caras y el consenso entre varones de diferente procedencia (asiáticos, africanos, chinos, hindúes, ingleses, estadounidenses) en cuáles eran las mujeres más guapas y cuáles eran las menos atractivas (Thakerar \& Iwawaki, 1979) sugieren que los componentes de la belleza no son arbitrarios ni están ligados a la cultura (véase también Buss, 1989; Cellerino, 2002; Cross \& Cross 1971; Langlois, Roggman \& Reiser-Danner, 1990). Evidencias a favor de una base biológica más que cultural en la valoración del atractivo facial aportaron Langlois y cols. (1987) en un estudio realizado con niños de 2 a 8 meses de edad a los que se les mostró una serie de imágenes de caras femeninas en las que el atractivo facial había sido valorado previamente por adultos. Los pequeños observaron por más tiempo la cara más atractiva, lo que indica que desde los dos meses de edad existen preferencias similares a las de los adultos.

Los estudios realizados sobre la simetría facial han demostrado que el grado de asimetría puede ser un indicador importante de estabilidad del desarrollo y un biomarcador de salud (Jones, Little, Penton-Voak, Tiddeman, Burt, \& Perrett, 2001). La asimetría fluctuante (AF) es el estado más frecuente en el que no existe ninguna tendencia hacia un lado que tenga una mayor magnitud que la del opuesto. Se trata más bien de desviaciones sutiles y aleatorias de la simetría de rasgos bilateralmente simétricos, en el que la media de la diferencia entre la medida del lado derecho e izquierdo no difiere estadísticamente de cero (Van Valen, 1962). Dentro de los factores que incrementan el nivel de AF se encuentran factores extrínsecos 
como el estrés térmico, el estrés audiogénico, presión parasitaria, aumento en la densidad de la población, altas concentraciones de sustancias químicas como el mercurio, níquel, DDT, hidrocarburos aromáticos y derivados de compuestos xenoestrogénicos (Møller \& Swaddle, 1997), y los factores intrínsecos que se encuentran asociados a perturbaciones genéticas debidas a la endogamia. En consecuencia, la simetría bilateral de ciertos parámetros faciales ha sido planteada como el reflejo de un desarrollo de buena calidad, especialmente en la capacidad de resistir a las perturbaciones ambientales durante las fases más sensibles del desarrollo. También se ha correlacionado con la heterocigosidad y la resistencia parasitaria (Thornhill \& Gangestad, 1993) de manera que una cara simétrica con baja AF puede indicar la capacidad de un individuo de hacer frente a los retos de su ambiente y estar correlacionada con la adaptabilidad (Downes, 2005).

Diversos estudios indican que los hombres más simétricos, es decir, con baja AF tienen mayor éxito de emparejamiento, por ejemplo, un alto número de parejas sexuales, una edad de inicio sexual más temprana y un acceso sexual a una nueva pareja más rápido (Baker, 1997; Gangestad \& Thornhill, 1977; Thornhill \& Gangestad, 1994 citado en Thornhill \& Gangestad, 1999). La baja AF en hombres más simétricos parece ser un rasgo predictor de una frecuencia relativamente alta de los orgasmos de cópula en sus parejas sexuales (Thornhill, Gangestad \& Comer, 1995).

\section{Asimetría fluctuante y cambios durante el ciclo menstrual}

Una de las fases más particulares en el ciclo menstrual de la mujer es la fase ovulatoria, etapa potencialmente fértil donde, en relación a otras fases del ciclo, se han evidenciado cambios en la elección de una pareja, así como en la posibilidad de ser elegidas. Se ha demostrado (Manning, et al., 2002; Manning, et al., 1996, citado en Grammer, Moller, Fink, \& Manning, 2005) que la asimetría fluctuante (AF) puede variar de manera cíclica durante el ciclo menstrual de las mujeres, encontrándose los valores más bajos durante la ovulación. También varían las preferencias de las mujeres durante el ciclo menstrual al valorar el atractivo facial masculino (Thornhill \& Gangestad, 1999). Durante el ciclo menstrual también varía el interés sexual de las mujeres. Sus deseos sexuales y el índice de cópulas extrapareja (CEP) presentan los mayores valores durante la fase medio folicular y 
de ovulación, momento en el que la fertilidad de la mujer y la probabilidad de quedar embarazada es mayor (Baker \& Bellis, 1995). En el estudio realizado por Jasienska, Lipson, Ellison, Thune \& Ziomkiwicz (2006) se midieron los niveles de estradiol a lo largo del ciclo menstrual en un grupo de 171 mujeres. Los resultados arrojaron que los niveles de estradiol a mediados del ciclo en las mujeres simétricas fue casi un 30\% más alto que aquellos en las mujeres asimétricas de la misma población. La diferencia en los niveles hormonales sugiere un incremento en la probabilidad de concepción en mujeres simétricas. En resumen, la alta simetría de ciertos parámetros faciales se ha planteado como el reflejo de un desarrollo de buena calidad, por lo que individuos simétricos podrían tener una ventaja por sobre competidores asimétricos durante la elección de pareja (Møller \& Swaddle, 1997).

A partir de lo anterior, el objetivo de nuestro estudio fue analizar la importancia de la simetría facial en la evaluación de distintos atributos en la elección de una potencial pareja del sexo contrario. Asimismo, consideramos importante investigar si existían diferencias entre varones y mujeres, y si en ellas la fase del ciclo menstrual influía en dicha elección.

\section{Método}

Se aplicaron 758 encuestas en diversos espacios públicos de la región Metropolitana de Santiago de Chile, de las cuales se consideraron 718, que corresponde al 95\% que manifestó ser heterosexual. El 5\% restante que manifestó una tendencia homosexual y bisexual no fueron incluidos en este estudio. De las 718 personas encuestadas que manifestaron ser heterosexuales, 262 encuestados (36\% del total de la muestra) eran varones (promedio de edad: 30 años; rango: $18-62$ años; $\left.{ }^{2} * \mathrm{DS}: 11\right)$ y 456 encuestados (64\% del total de la muestra) eran mujeres (promedio de edad: 29 años; rango: 18-63 años; DS: 9,6).

La encuesta fue aplicada en lugares con gran afluencia de público de la Región Metropolitana, como estaciones de metro, universidades y centros comerciales, durante diez días. A todos los individuos que aceptaron responder la encuesta se les informó que se trataba de un estudio sobre elección de pareja, además de recalcar que la encuesta era de carácter anónimo.

$2 *$ DS = Desviación Standard. 
A cada individuo se le entregó una encuesta, un lápiz y un set con tres imágenes de hombres y tres imágenes de mujeres, las cuales se encontraban rotuladas con las letras T, G y M, letras que no fueron consecutivas para no influenciar la elección del encuestado. A cada persona se le explicó cómo debía rellenar la encuesta: en la primera parte debían completar sus datos personales, y en la segunda parte debían responder, observando el set de imágenes T, G y M, de acuerdo al atributo solicitado en la tabla para una posible relación. Los atributos que debían evaluar fueron: atractivo, personalidad, suerte con el sexo opuesto, inteligencia, salud, deseabilidad por una relación a corto y largo plazo, mayor cuidado de los hijos y dominancia social. Para el caso de las mujeres encuestadas debían completar además un recuadro donde indicaban el primer día de su última regla. A partir de esta información obtuvimos la fase del ciclo en la que se encontraban. Dentro del ciclo menstrual se consideró la fase menstrual (días 1-5), fase folicular (días 6-12), fase ovulatoria (días 13-15) y fase lútea (días 16-28).

\section{Sujetos fotografiados}

Se fotografió a 50 sujetos hombres y mujeres entre los 22 y 25 años, se les explicó que las imágenes sólo serían utilizadas con fines de investigación. De las 50 imágenes se descartaron las que presentaban exceso de luz, sombras y objetos que distrajeran al observador. Además se estandarizaron las variables como el color de los ojos, el color de cabello y el color de piel. De esta forma se eligieron las tres mejores fotografías de hombres y de mujeres.

\section{Obtención de las fotografias}

Las fotos fueron tomadas con máquina fotográfica digital Olympus modelo SP-550UZ de 7,1 megapixeles con un zoom de 18X óptico, tamaño de 3.072 X 2304, con una calidad de compresión Super Hi Quality, lo cual generaba fotos de 3,5 megapixeles aproximadamente. Todos los registros fueron hechos a una distancia de dos metros, minimizando así los efectos de distorsión por acercamiento (distorsión por amplitud).

Se cauteló que ambos pabellones auriculares se viesen en igual magnitud para confirmar un registro ortográfico con el plano medio sagital de los individuos. 
El punto central de foco se dirigió a la zona subnasal. Antes de sacar la fotografía se les solicitó a los individuos mantener una expresión facial neutra con labios relajados, además de no usar pendientes ni colgantes.

Las medidas de los parámetros faciales se realizaron en editor de imágenes Adobe Photo Shop versión CS-2.

\section{Orientación del archivo fotográfico}

Para realizar el análisis de simetría facial fue necesario hacer coincidir el plano medio sagital con la vertical verdadera que se refleja en la pantalla del monitor. Para ello se horizontalizó, a través de un programa, la línea bipupilar, considerando el punto medio entre ambas pupilas. Sobre la misma línea se trazó una guía simetral a ésta que representaría el plano medio sagital. De esta manera se analizaron las estructuras medianas y paramedianas para establecer la simetría horizontal y vertical.

En las siguientes imágenes se establecen los 5 parámetros absolutos para la simetría horizontal y vertical.

\section{Simetría Horizontal}

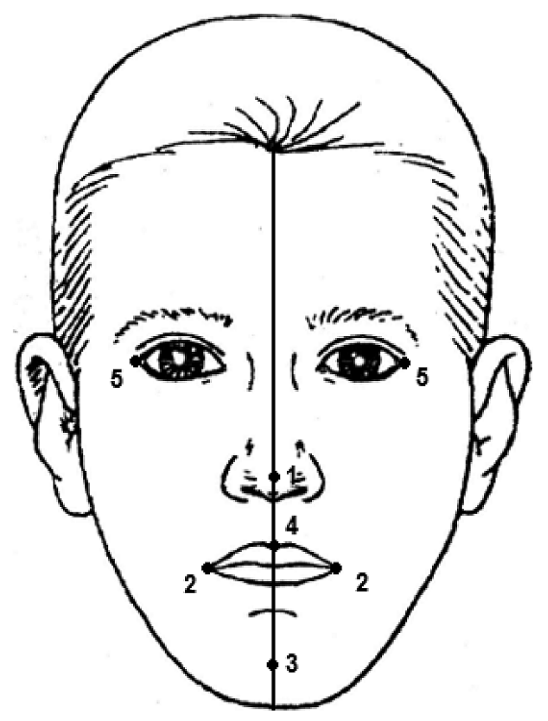

1. Pirámide nasal

2. Comisura labial

3. Línea media del mentón

4. Parte media arco de cupido

5. Ángulo externo del ojo

(Imagen: Gutiérrez, 2004) 


\section{Simetría Vertical}

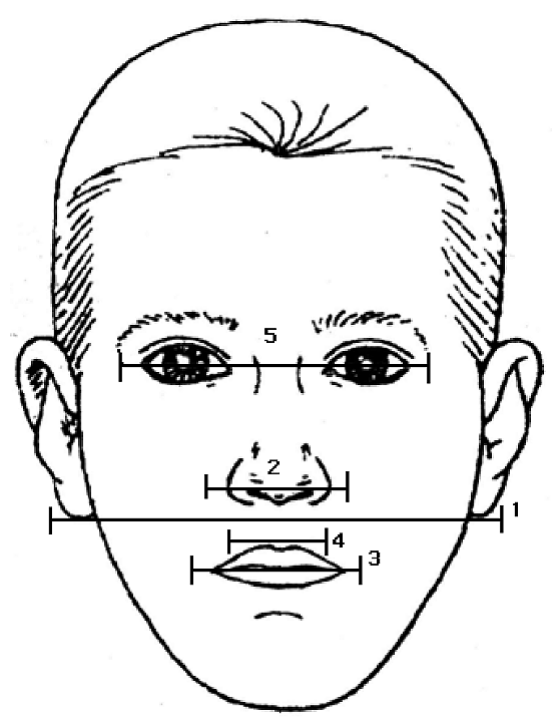

1. Lóbulo de la oreja

2. Borde superior de las narinas

3. Comisura labial

4. Arco de cupido

5. Ángulo externo del ojo

(Imagen: Gutiérrez, 2004)

\section{Mediciones del registro fotográfico}

\section{Simetría horizontal}

Puntos medianos: ubicando el sistema de referencia para mediciones del programa coincidente con el plano mediosagital establecido se procedió a determinar el grado de desviación lateral; este valor fue restado matemáticamente a un valor 10 fijado arbitrariamente, de esta manera se obtuvo un valor menor a 10 que representaría el lado hacia el cual estaría desviada la estructura medial, considerándose el otro lado como "normal" por lo cual se le asignaría el valor 10 anteriormente mencionado.

Estructuras paramedianas: se consignó la distancia de estas estructuras al plano medio sagital.

\section{Simetría vertical}

Esta simetría consideró sólo estructuras paramedianas: el operador eligiendo arbitrariamente uno u otro lado trazó una guía simetral al plano medio sagital, que pasando exactamente por el punto o estructura de un 
lado se midió el grado de desplazamiento hacia arriba o hacia abajo de la estructura contralateral (de la misma estructura del otro lado). A la estructura del lado usado como referencia se le asignó arbitrariamente un valor 10 y la estructura contralateral un valor de 10 menos el grado de desplazamiento vertical registrado.

\section{Determinación del grado de simetría facial}

Para cada estructura estudiada fueron consignados dos valores. El valor de simetría para cada estructura estudiada estuvo dado por el porcentaje que representó el valor menor respecto del valor mayor. Se determinaron las asimetrías horizontales para todos los individuos estableciéndose un valor que correspondió al promedio de los parámetros estudiados; de igual manera se hizo para la simetría vertical. De esta manera, se obtuvo un valor promedio de simetría horizontal y vertical para cada individuo y el valor promedio de estas dos correspondió al valor de simetría total. Para los análisis estadísticos las imágenes antes mencionadas como T, G y M se modificaron por S, SS y SSS respectivamente. La imagen S corresponde a la imagen con menor simetría facial, SS la imagen medianamente simétrica y SSS la imagen con mayor simetría facial. Los índices de simetría facial (calculados a partir de la simetría vertical y horizontal) de las mujeres fueron: $S=96,28$; $S S=96,93$; $S S S=98,47$. Los índices de simetría de los varones fueron: $S=98,11 ; S S=98,56 ; S S S=99,08$.

\section{Análisis estadísticos}

Mediante el software SPSS versión 15.0 se realizaron análisis de frecuencias para la estadística descriptiva de los datos y se utilizó la prueba de Chi 2 para contrastar si las diferencias observadas coincidían con las frecuencias esperadas para cada variable dependiente analizada.

\section{Resultados}

Evaluación, por parte de los hombres, de los rostros femeninos

Los hombres mostraron una tendencia hacia la elección de la imagen con menor simetría $(S)$ facial, que fue percibida como la mujer 
con más personalidad, más suerte con el sexo opuesto, más saludable, más deseable para una relación sexual y dominante socialmente. Sin embargo, los atributos que mostraron diferencias significativas fueron suerte con el sexo opuesto $\left(\mathrm{X}^{2}=5,44 ; \mathrm{p}=0,020\right)$ y la dominancia social $\left(\mathrm{X}^{2}=7,20 ; \mathrm{p}=0,007\right)$. No así las variables personalidad $\left(X^{2}=1,04 ; p=0,306\right)$, salud $\left(X^{2}=1,63\right.$; $\mathrm{p}=0,201)$ y deseable para una relación sexual $\left(\mathrm{X}^{2}=0,18 ; \mathrm{p}=0,66\right)$, las cuales no mostraron diferencias significativas (Figura 1). Ambas imágenes, la más simétrica (SSS) y la menos simétrica (S), fueron consideradas por los hombres como las más atractivas, pero ninguna de ellas presentó diferencias significativas. Para las variables inteligencia, deseabilidad para una relación a largo plazo y cuidado de los hijos, los hombres se inclinaron por la mujer con mayor simetría facial (SSS), sin embargo, en ninguno de estos atributos se encontraron diferencias significativas.

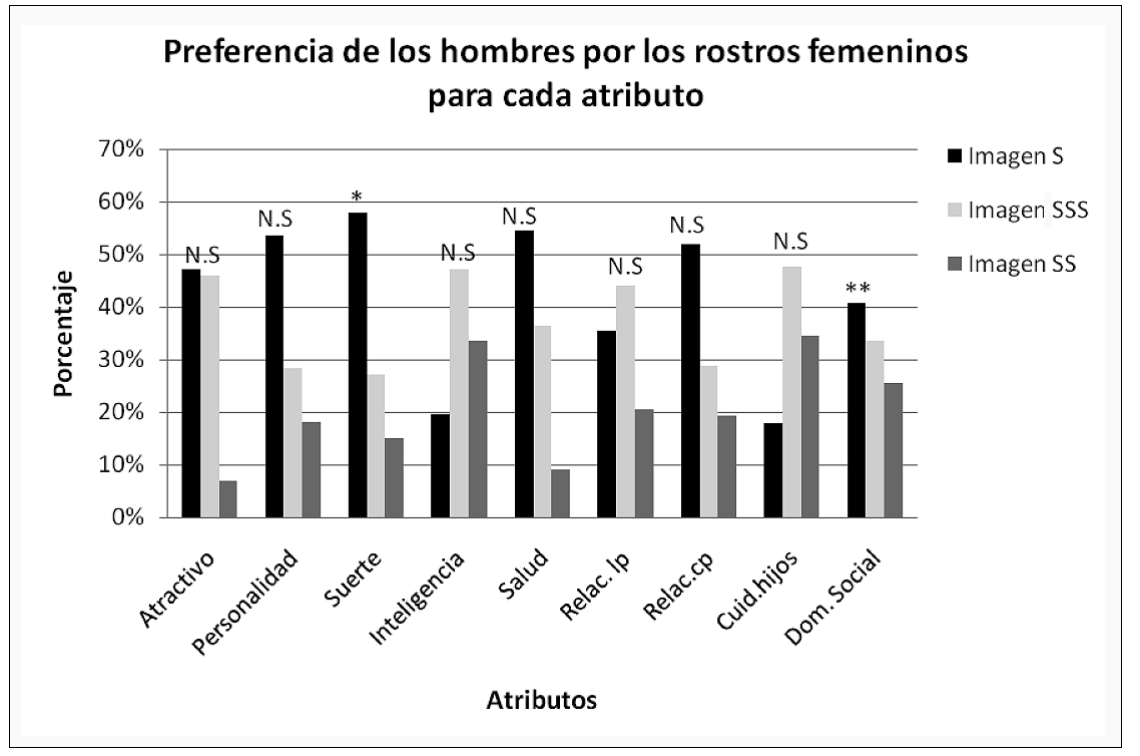

Figura 1. Preferencias de los hombres por los rostros femeninos para cada atributo. Los niveles de significatividad estadística son: N.S para $\mathrm{p}>0.05$; $^{*} \mathrm{p}<0.05$; ${ }^{* *} \mathrm{p}<0.01$.

Evaluación, por parte de las mujeres, de los rostros masculinos

Las mujeres seleccionaron de manera significativa al hombre con mayor simetría facial (SSS) percibiéndolo como más atractivo $\left(\mathrm{X}^{2}=89,36\right.$; 
$\mathrm{p}=0,000)$, mayor personalidad $\left(\mathrm{X}^{2}=55,60 ; \mathrm{p}=0,000\right)$, más suerte con el sexo opuesto $\left(X^{2}=100,52 ; p=0,000\right)$, más saludable $\left(X^{2}=6,98 ; p=0,008\right)$, más deseable para una relación estable $\left(\mathrm{X}^{2}=7,77 ; \mathrm{p}=0,005\right)$ y para una relación sexual $\left(\mathrm{X}^{2}=33,55 ; \mathrm{p}=0,000\right)$. Sin embargo, el hombre con menor simetría facial $(S)$ fue percibido significativamente como más inteligente $\left(\mathrm{X}^{2}=17,88 ; \mathrm{p}=0,000\right)$ (Figura 2). Para la variable cuidado de los hijos las preferencias por la imagen con menor simetría facial no fueron significativas $\left(\mathrm{X}^{2}=0,01 ; \mathrm{p}=0,917\right)$, como tampoco lo fueron para la dominancia social las preferencias por la imagen más simétrica (SSS) $\left(\mathrm{X}^{2}=2,96 ; \mathrm{p}=0,085\right)$ y medianamente simétrica (SS) $\left(\mathrm{X}^{2}=3,70 ; \mathrm{p}=0,055\right)$.

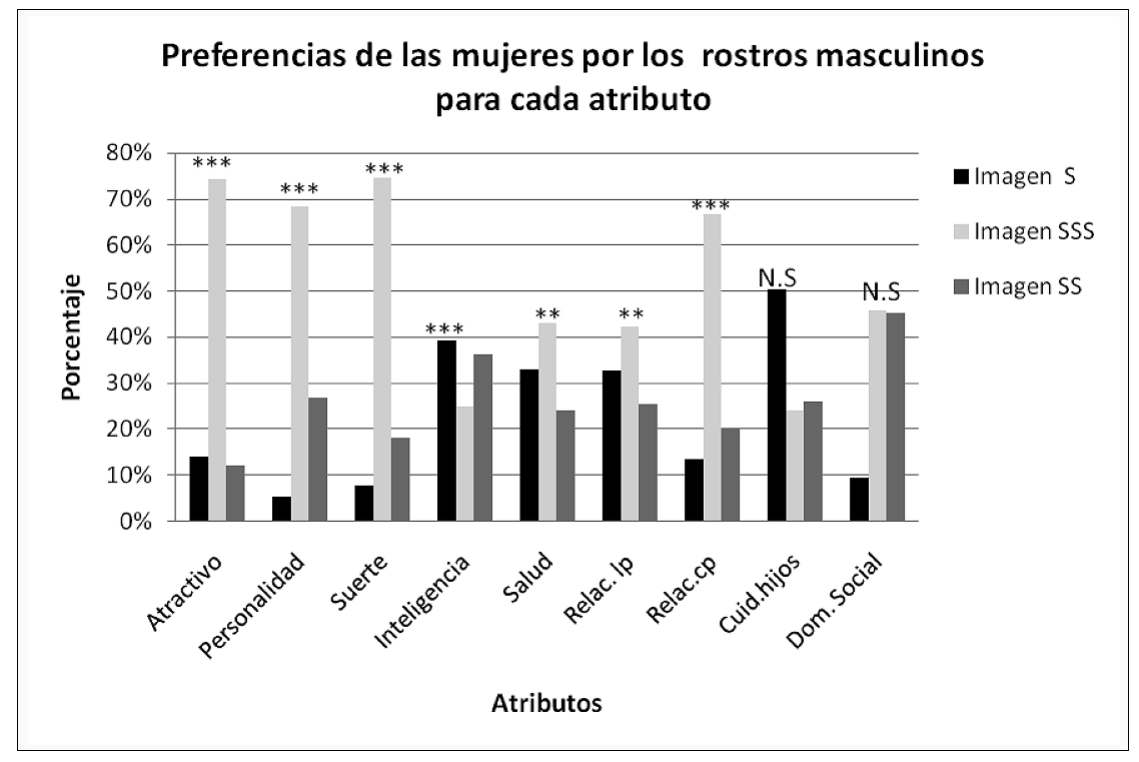

Figura 2. Preferencias de las mujeres por los rostros masculinos para cada atributo. Los niveles de significatividad estadística son: N.S para $\mathrm{p}>0.05$; $^{*} \mathrm{p}<0.05$; $^{* *} \mathrm{p}<0.01$; $^{* * *}$ $\mathrm{p}<0.001$

La evaluación de los rostros masculinos por parte de las mujeres en función de la fase del ciclo menstrual no presentó diferencias significativas en ninguno de los atributos evaluados. 


\section{Discusión}

Los resultados de nuestra investigación están de acuerdo con los de otros estudios en los que se concluye que la simetría facial es un factor que influye en la evaluación de los atributos en el proceso de la elección de una potencial pareja. Las variables consideradas en otras investigaciones sobre la simetría facial (e. g. Grammer \& Thornhill, 1994; Thornhill \& Gangestad, 1993 y 1999) han sido principalmente el atractivo físico, la salud, y las relacionadas con el éxito del emparejamiento (básicamente porque son las más relacionadas con la "calidad genética" y las alteraciones en el desarrollo). Además de esta concordancia con otros trabajos, nuestros resultados aportan información sobre la relación entre simetría facial y rasgos que hombres y mujeres buscan en una potencial pareja que han sido apenas considerados en otras investigaciones, tales como la inteligencia, la dominancia social y el cuidado de los hijos. Una conclusión importante de este estudio es que, tanto en hombres como en mujeres, la evaluación de la inteligencia y el cuidado de los hijos fueron contrarios a la evaluación del atractivo físico, salud y deseable para una relación sexual.

Los resultados arrojaron diferencias entre las respuestas de hombres y mujeres, observándose entre las respuestas femeninas una preferencia para la mayoría de las variables por el hombre más simétrico (SSS). Contrariamente a lo esperado, los hombres se inclinaron por la imagen de menor simetría facial (S). Estos resultados apoyan las predicciones evolutivas sobre las diferencias en la elección de pareja entre varones y mujeres, ya que las mujeres se mostraron más selectivas que los hombres del estudio.

En el caso de las mujeres, constatamos una preferencia significativa hacia la imagen con mayor simetría para los atributos de: atractivo, personalidad, suerte con el sexo opuesto, más saludable, deseable para una relación estable y deseable para una relación sexual a corto plazo (affaire o infidelidad). A partir de lo anterior nuestro estudio sugiere que las mujeres son capaces de detectar en una potencial pareja una baja asimetría fluctuante o bien una cara simétrica, evaluando atributos que consideran importantes para el éxito con una potencial pareja. La preferencia por el hombre con mayor simetría facial nos da cuenta de una preferencia de las mujeres por realizar un emparejamiento con hombres que posean "buenos genes" y así incrementar la viabilidad de la descendencia y el éxito reproductor. 
Dentro de las predicciones evolutivas, en relación a los rasgos buscados por las mujeres, se encuentra la colaboración en el cuidado de crías. En el presente estudio el atributo cuidado de los hijos, evaluado por las mujeres, no presentó diferencias con la simetría facial. Frente a ello es que creemos que la única forma en que las mujeres pueden evaluar este atributo en una potencial pareja es a través de la elección de hombres que sean "buenos proveedores", que en la actualidad se expresa con el buen nivel económico de los hombres.

En cuanto a la variable ciclo menstrual en las mujeres, nuestros resultados no presentaron diferencias significativas al evaluar los rostros con distinta simetría y por ello no son concordantes con los encontrados en otros estudios que indican que en fase ovulatoria las mujeres seleccionan hombres con mayor simetría facial. Frente a esto creemos que nuestra muestra, aun cuando fue amplia, las mujeres que se encontraban en fase ovulatoria en el momento de la consulta sólo representaban una pequeña parte del total de la muestra.

Los hombres se inclinaron, en la mayoría de los atributos, por la imagen con menor simetría facial (S); sólo en los atributos suerte con el sexo opuesto y dominancia social se encontraron diferencias significativas. La tendencia observada hacia la imagen de la mujer con menor simetría facial probablemente se pueda explicar porque esta mujer tenía una pintura labial casi imperceptible, pero que tal vez fue una característica de mayor preferencia para los hombres que la menor simetría facial. Este resultado pensamos que puede tener mucha importancia dado que los investigadores que trabajan en elección de pareja y en las explicaciones basadas en la biología evolutiva, por ejemplo la psicología evolucionista, sugieren que solamente influyen los mecanismos psicológicos (muchas veces de forma inconsciente) que permiten evaluar en la potencial pareja los atributos que confieran un mayor éxito reproductor. Gil-Burmann, Peláez \& Sánchez (2002) encontraron en su estudio sobre elección de pareja en la población española que los mecanismos psicológicos y la preferencia por determinados rasgos en la pareja pueden ser flexibles y contingentes con el ambiente y proponen la hipótesis de que la evolución hace posible cierta plasticidad de los individuos para ajustar y variar sus preferencias en respuesta a cambios sociales, económicos o culturales. La preferencia de los hombres chilenos encuestados en este trabajo por la mujer con los labios ligeramente pintados, pero con la menor simetría facial no indica que la simetría facial de las mujeres no 
influya en la elección de los hombres, sino que puede haber un rasgo artificial (en este caso un producto de belleza) que pueda ser una señal fenotípica más importante que la simetría facial. La utilización de productos de belleza, perfumes, pinturas, adornos, etc., por las mujeres para realzar su atractivo físico desde hace miles de años probablemente sea un mecanismo psicológico que ha contribuido a aumentar su eficacia biológica y no debe ser ignorado por los científicos que trabajan dentro del marco de la biología evolutiva.

La simetría facial es, y sigue siendo, una veta en la que varios investigadores se han sumergido con trabajos que han arrojado resultados sorprendentes de la selección sexual en humanos. Un aspecto importante a destacar es la metodología utilizada en nuestro estudio para medir la simetría facial, donde fueron utilizados los valores de cinco parámetros verticales y cinco parámetros horizontales, obteniéndose a partir de ello un índice de la simetría facial. Los índices de simetría facial en la mayoría de los estudios sólo utiliza la simetría horizontal, proporcionando medidas de simetría menos rigurosas que cuando se calcula también la simetría vertical.

\section{Referencias bibliográficas}

Baker, R. R. \& Bellis, M. A. (1995). Human Sperm Competition: Copulation, Masturbation and Infidelity. London: Chapman and Hall.

Buss, D. M. (1989). Sex differences in human mate preferences: Evolutionay hypotheses tested in 37 cultures. Behavioral and Brain Sciences, 12, 1-49.

Cellerino, A. (2002). Facial attractiveness and species recognition: an elementary deduction? Ethology, Ecology and Evolution, 14, 227-237.

Cross, J.F. \& Cross, J. (1971). Age, sex, race, and the reception of facial beauty. Developmental Pychology, 5, 433-439.

DARWin, C. (I 87I). The descent of man and selection in relation to sex. Murray: Londres. (El Origen del Hombre y la Selección en Relación al Sexo, 1982).

Downes, S. (2005). Integrating the multiple biological causes of human behavior. Biology and Philosophy, 20, 177-190.

Gil-Burmann, C., Peláez, F. \& Sánchez, S. (2002). Mate choice differences according to sex and age: an analysis of personal advertisements in Spanish newspapers. Human Nature, 13, 493-508.

Grammer, K. \& Thornhill, R. (1994). Human (Homo sapiens) facial attractiveness and sexual selection: The role of symmetry and averageness. Journal of Comparative Psychology, 108(3), 233-242. 
Grammer, K., Moller, A., Fink, B. \& Manning, J. (2005). Physical attractiveness and health: Comment on Weeden and Sabini (2005). American Psychological Association, 131(3), 658-661.

Gutiérrez, R. (2004). Estudio de la relación entre características antropométricas, atractivo facial y calidad seminal. Tesis doctoral, Universidad de Valencia. España.

Jasienska, G., Lipson, S., Ellison, P., Thune, I. \& Ziomkiwicz, A. (2006). Symmetrical women have higher potential fertility. Evolution and Human Behavior, 27, 390-400.

Jones, B. C., Little, A. C., Penton-Voak, I. S., Tiddeman, B.P., Burt, D. M. \& Perrett, D. I. (2001). Facial symmetry and judgements of apparent health support for a "good genes" explanation of the attractiveness-symmetry relationship. Evolution and Human Behavior, 22, 417-429.

Langlois, J. H., Roggman, L. A., Casey, R. J., Ritter, J. M., RieserDAnner, L. A. \& Jenkins, V. Y. (1987). Infant preferences for attractive faces: rudiments of a stereotype? Developmental Psychology, 23, 363-369.

Langlois, J. H., Roggman, L. A. y Reiser-Danner, L. A. (1990). Infants' differential social responses to attractive and unattractive faces. Developmental Psychology, 26, 153-159.

Møller, A. P. \& Swadle, J. P. (1997). Asymmetry, Developmental Stability and Evolution. New York: Oxford University Press.

Thakerar, J. N. \& Iwawaki, S. (1979). Cross-cultural comparisons of interpersonal attraction of females toward males. Journal of Social Psychology, 108, 121-122.

Thornhill, R. \& Gangestad, S. (1993). Human facial beauty: averageness, symmetry and parasite resistance. Human Nature, 4, 237-269.

Thornhill, R., Gangestad, S. \& Comer, R. (1995). Human female orgasm and mate fluctuating asymmetry. Animal Behavior, 50, 1601-1615.

Thornhill, R. \& Gangestad, S. (1999). The scent of symmetry: A human sex pheromone that signals fitness? Evolution and Human Behavior, $20,175-201$.

Van Valen, L. (1962). A study of fluctuating asymmetry. Evolution, 16, $125-142$.

Fecha Recepción de artículo: 25 de agosto de 2009.

Fecha Aceptación de artículo: 09 de noviembre de 2009. 